\title{
PTolemy - Towards Cosmological Relic Neutrino detection
}

\section{Alfredo G. Cocco*}

Istituto Nazionale di Fisica Nucleare (Italy)

E-mail: alfredo.cocco@na.infn.it

The PTolemy project aim at the direct detection of the Cosmological Relic Neutrino background by the use of a Tritium target. Cosmological Relic Neutrino produced in the early stage of the Big Bang are predicted to have thermally decoupled from other forms of matter at approximately 1 second after the Big Bang; they represent the oldest detectable Big Bang relics and as such they carry an invaluable content of information about the genesis and evolution of our Universe. Despite their very small energy they present a sizable interaction cross section on nuclei that decay through beta decay, as it was pointed out by recent studies. In particular Tritium is among the nuclei having the most favorable detection conditions. We will report here the status of construction of the PTolemy prototype being built in order to demonstrate the key aspects of the detection technique.

Neutrino Oscillation Workshop

4 - 11 September, 2016

Otranto (Lecce, Italy)

\footnotetext{
* Speaker.
} 


\section{Introduction}

According to the Big Bang model light neutrinos produced in the early universe are predicted to have thermally decoupled from other forms of matter at approximately 1 second after the Big Bang. The light neutrinos, believed to be stable particles, have cooled to a temperature of $1.9 \mathrm{~K}$ $\left(1.7 \times 10^{-4} \mathrm{eV}\right)$ in the present day and are predicted to have an average number of density of approximately $56 / \mathrm{cm}^{3}$ per lepton flavor. The number density prediction is based on the annihilation rate of $\mathrm{e}^{+} \mathrm{e}^{-}$into three flavors of neutrinos through the weak neutral current interaction in the dense, high temperature conditions of the early universe.

At present, the direct measurements on the lightest neutrino mass eigenstate give results in the order of the eV. Oscillation experiments only provide a lower limit on the mass of (at least) one neutrino mass eigenstate of the order of $0.05 \mathrm{eV}$, while direct measurements of the electron energy spectrum in ${ }^{3} \mathrm{H}$ decay give $m_{v}<2 \mathrm{eV}$ [1]. A large improvement in this respect will be provided by the KATRIN experiment, whose expected sensitivity is $0.2 \mathrm{eV}$. On the other hand, cosmological data from cosmic microwave background anisotropies and the large scale structure power spectrum provide an independent bound on the sum of neutrino masses which, depending on the particular model adopted and the number of free parameters, lies in the range $0.3 \div 2 \mathrm{eV}$; see e.g. [2]. As we will argue in the following, if $m_{v}$ is in the $0.1 \div 1.0 \mathrm{eV}$ range, the PTolemy experiment may represent the unique way to detect cosmological relic neutrinos.

The idea of using Neutrino Capture on Beta decaying nuclei to measure the cosmological relic neutrino background predicted in the framework of the hot big bang model was recently pointed out in $[3,4]$; in these papers authors make an extensive study of the threshold-less reaction, of its cross section and on the effects of the recently discovered neutrino oscillations on the possible detection of very low energy neutrino. They found that a Tritium target mass of 100 grams is predicted to produce approximately 10 events/year from relic neutrino capture with the relic neutrino density modeled as a uniform Fermi-Dirac number density throughout space. The local neutrino density may be enhanced in galactic clusters by factors that range from 1-100 depending on the neutrino mass [5]. The uncertainty on the neutrino capture cross section on tritium is constrained to the subpercent level, and among the possible nuclei unstable to $\beta$-decay the tritium nucleus is considered optimal based on the product of the capture cross section and the half-life of 12.3 years uniquely coming from the $\beta$-decay process.

The PTolemy experiment [6] aim at the detection of cosmological relic neutrino background using a 100 grams tritium target and leading edge technology in order to achieve an unprecedented discrimination power of signal events with respect to the overwhelming amount of electrons produced in the $\beta$-decay. Similarly, PTolemy is capable of reaching the highest sensitivity in the search for $\mathrm{keV}$-scale dark matter candidates in the form of sterile neutrinos with an admixture of electron flavor at the level of $\left|U_{e 4}\right|^{2}$ of $10^{-4} \div 10^{-6}$, depending on the sterile neutrino mass.

\section{The PTolemy experiment prototype}

The PTolemy experiment conceptual design is described in Figure 1; the electrons produced in the beta decay and neutrino capture process of the $100 \mathrm{~g}$ Tritium target are focused to the bore of a MAC-E filter where a first stage of background event rejection is performed. An ideal cut on the 
last $\mathrm{eV}$ of electron beta spectrum would give an event rate reduction of about $1.55 \times 10^{-13}$. The surviving electrons are then accelerated by an electric field and focused into an RF tracking device able to provide both energy measurement and time-of-flight tag for the downstream micro calorimeter. The electron is then de-accelerated to about $100 \mathrm{eV}$ where a cryogenic micro-calorimeter can measure the energy with an accuracy of about $0.1 \mathrm{eV}$.

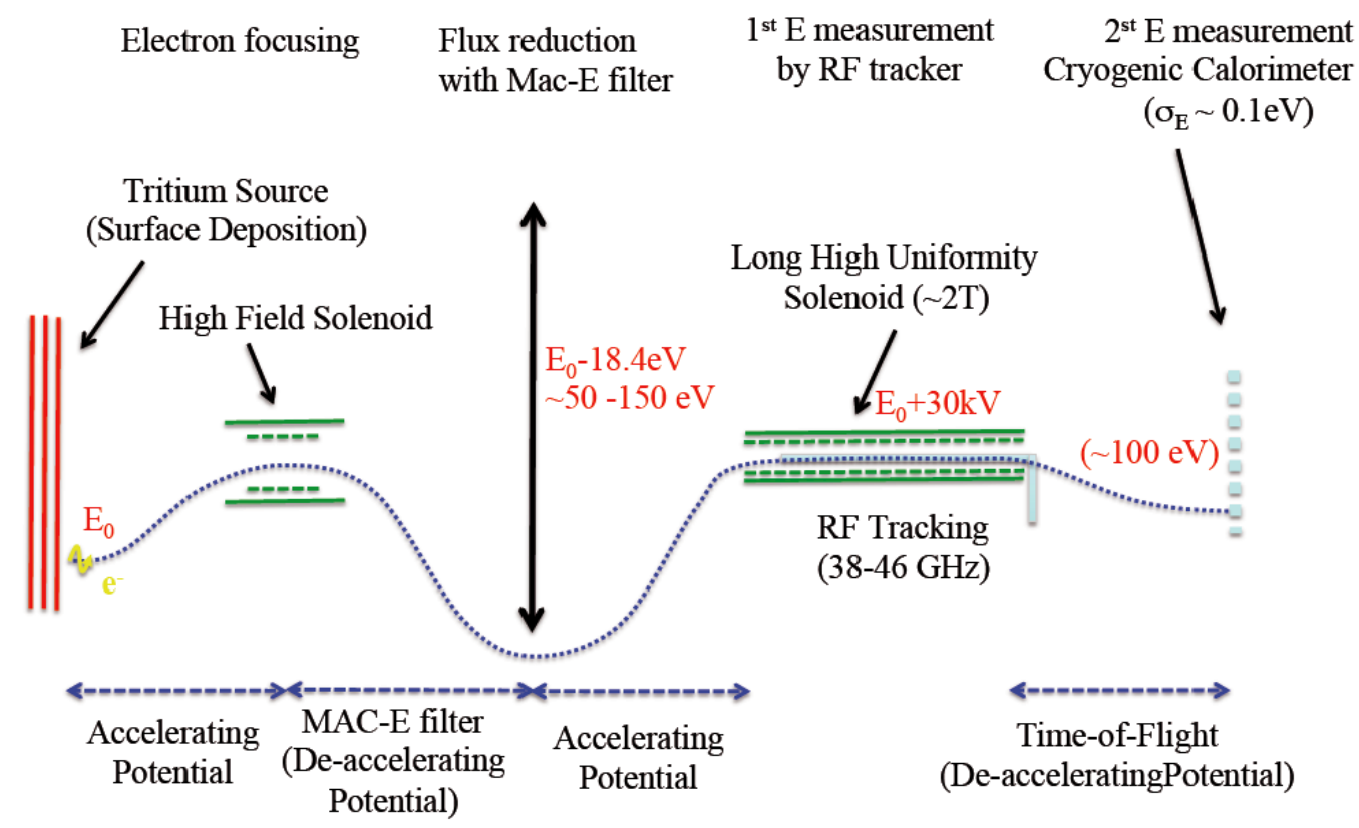

Figure 1: Schematic description of the PTolemy experiment. The prototype is under construction at the Princeton Plasma Physics Laboratory (PPPL). First results are expected by the first half of 2017.

The PTolemy experiment is based on an atomic thickness tritium target. The large surface area required for relic neutrino detector can be factorized into isolated tritium target areas using a method of magnetic ducts. Half of the $4 \pi$ solid angle of the isotropically emitted electrons from a planar Tritium target are accelerated with a precision voltage reference into a high magnetic field region at the opening of the MAC-E filter. Graphene substrates are suitable to hold monoatomic Tritium layers through chemical absorption. The binding energy of tritium on graphene is an order of magnitude weaker than the $\mathrm{T}_{2}$ binding energy [7]. The differential density of final states of the ${ }^{3} \mathrm{He}$ is expected to be reduced by an order of magnitude. By binding the tritium to individual layers of graphene, the high conductivity of graphene is also expected to eliminate the effect of charging and voltage reference shifting. The PPPL site has already demonstrated the ability to safely handle 100 grams of tritium during the operation of the Tokamak Fusion Test Reactor (TFTR). PPPL Tritium Facility, located at D-Site, contains the appropriate equipment for supporting prototype, mid-range, and full scale PTolemy configurations. The facility is fully instrumented for storage, purication, analysis, delivery, and recovery of tritium.

MAC-E filter in PTolemy acts as an integrating high-pass filter. The use of a detector with precision calorimetry removes the need for a sharp cutoff; the main role of the MAC-E filter is to remove enough of the spectrum below the endpoint so that the detector may function without being swamped by large signal rates. 
Tritium decay endpoint electrons have energy of about $18.6 \mathrm{keV}$, corresponding to a total velocity of approximately $\beta=0.26$. For $\mathrm{B}=1.9 \mathrm{~T}$, the value of the cyclotron frequency is of approximately $46 \mathrm{GHz}$. An endpoint electron moving transversely to the magnetic field will radiate approximately $P_{t o t}=3 \times 10^{-14} \mathrm{~W}$ of coherent RF power. Single electron measurement in $\mathrm{RF}$ trackers has been recently proven as a viable technology in Project- 8 prototypes. Thread electron trajectories through an array of such antennas with wide bandwidth can be used to identify cyclotron RF signal in transit times of order $0.2 \mathrm{msec}$. The timing resolution is expected to be of the order of $10 \mathrm{~ns}$, depending on micro-calorimeter response.

Electron calorimeter should have an energy resolution sufficient to resolve the neutrino mass. Recent development of TES cryogenic micro calorimeters may prove the feasibility of having a resolution of $0.1 \mathrm{eV}$ in the measure of the electron energy. In case of the Tritium decay endpoint decelerated to the $10 \div 100 \mathrm{eV}$ energy window the electron can be stopped with a very thin absorber (much thinner then the one used in X-ray measurements) thus reducing significantly the detector heat capacity. This has also a beneficial effect on the detector time response reaching bandwidths of about $1 \mathrm{MHz}$ to record $10 \mathrm{kHz}$ of electrons hitting the individual sensors. However, operating TES in magnetic field is a major technical challenge. The design for PTolemy incorporates magnetic shielding for the TES and the microwave-readout massive SQUID multiplexer (MMSM). Resolution of about $0.55 \mathrm{eV}$ at $1 \mathrm{keV}$ and of about $0.15 \mathrm{eV}$ at $0.1 \mathrm{keV}$ operating at $70 \div 100 \mathrm{mK}$ are under investigation.

The construction of the PTolemy prototype is almost completed [8]; in particular the first sample of the Tritiated graphene target is available and a complete proof-of-concept test with electron tracking from the target through the MAC-E filter to the endpoint TES calorimeter is planned for the beginning 2017. The PTolemy Collaboration foresee the collection of a large sample of tritium endpoint events using cryogenically cooled tritium and TES calorimeter; this may allow to verify that obtainable energy resolution is much better than that coming from diatomic tritium. Moreover, the most precise study of the tritium endpoint spectrum will be performed. Besides being a technology demonstrator the PTolemy experiment prototype may also provide interesting clues on the existence of sterile neutrino in the $\mathrm{keV}$ mass range.

\section{References}

[1] C. Patrignani et al. (Particle Data Group), Chin. Phys. C 40, 100001 (2016)

[2] J. Lesgourgues and S. Pastor, Phys. Rep. 429, 307 (2006)

[3] A.G. Cocco, G. Mangano and M. Messina, J. Cosmol. Astropart. Phys. 06, 015 (2007)

[4] A.G. Cocco, G. Mangano and M. Messina, Phys. Rev. D 79, 053009 (2009)

[5] A. Ringwald and Y.Y.Y. Wong, J. Cosmol. Astropart. Phys. 12, 005 (2004)

[6] S. Betts et al., [arXiv:1307.4738v2]

[7] C. Lin et al., Nano Lett. 15, 903 (2015)

[8] C. Tully, https://indico.cern.ch/event/432527/contributions/1071681 